\title{
Novel Methodologies for Food Quality and Provenance Fingerprints Assessment
}

\author{
Xavier Cetó $\left(D,{ }^{1}\right.$ José M. Díaz-Cruz, ${ }^{1,2}$ Figen Tokatli, ${ }^{3}$ Paolo Lucci, ${ }^{4}$ and Sabrina Moret ${ }^{4}$ \\ ${ }^{1}$ Department of Chemical Engineering and Analytical Chemistry, Faculty of Chemistry, University of Barcelona, Barcelona, Spain \\ ${ }^{2}$ Water Research Institute, Barcelona, Spain \\ ${ }^{3}$ Department of Food Engineering, Izmir Institute of Technology, Izmir, Turkey \\ ${ }^{4}$ Department of Agri-Food, Animal and Environmental Sciences, University of Udine, Udine, Italy
}

Correspondence should be addressed to Xavier Cetó; xavier.ceto@ub.edu

Received 19 March 2019; Accepted 19 March 2019; Published 17 April 2019

Copyright ( $\odot 2019$ Xavier Cetó et al. This is an open access article distributed under the Creative Commons Attribution License, which permits unrestricted use, distribution, and reproduction in any medium, provided the original work is properly cited.

The development of novel reliable methodologies that allow the control, assessment, and prediction of the characteristics of food products is a field under expansion nowadays, especially those that allow their characterization, classification, and authentication.

On the one side, the highly competitive global environment in food industry requires continuous innovation and a better sustainable usage of our natural resources in order to improve the high standards of food producers, leading to high value-added products. The linkage of new research ideas with food production provides a competitive advantage to food makers to fulfil the competitive market challenges.

On the other side, industry is increasingly interested on fast-response and low-cost methods to be used for the detection of adulterations or contamination of the products (either during or after its production) or to assess whether they guarantee quality control standards.

Food authentication is a multidisciplinary field that has input from instrumentation, biology, informatics, mathematics and statistics, agriculture, economics, and food technology. Current approaches for quality control are shifting from compound-oriented to pattern-oriented strategies, the former focusing on the identification of few specific compounds, while the latter targeting at the extraction of a global fingerprint of all the compounds present in the sample. Thus, fingerprinting techniques require the use of an analytical method that can simultaneously detect a large spectrum of compounds and provide comprehensive information of the sample, in combination with chemometric tools that allow the interpretation and extraction of meaningful data from the complex readings.

In this regards, this special issue has risen with the idea to cover advances in fast, reliable, and affordable characterization, classification and authentication of food products, and methodologies devoted to improve their quality.

When assessing food quality, perception of such foods by consumers is as critical as can be the identification of bioactive compounds or the detection of contaminations and/or adulterations. Characterization of food by analytical means can help on the comprehension of the chemical process that affects foods' physical characteristics and, consequently, consumers' quality perception. In this line, L. Yang evaluated the synergistic effect of trisodium citrate and microbial transglutaminase treatment on the textural properties of acidified yak skim milk gels. Results of the study allowed identification of the conditions that led to gels with higher stiffness, water holding capacity, and storage modulus.

In another subject, proteomic analysis has also gained an increasing role in the authentication of food products over the last years. Usage of proteins as markers complements traditional methodologies in the verification of the claims made about certain food products. To this aim, sample preparation and protein extraction is a key step in proteomic analysis so as to maximize protein recovery and minimize proteolysis and modification. In this regards, the contribution of A. della Malva et al. provide a comparison between 
two methods for the extraction and separation of myofibrillar proteins including solutions with different ionic strength in meat and fish muscles. The authors show how both extraction methods provided good solubilisation of myofibrillar proteins and derived fragments, with the denaturing solution leading to a more complex profile in terms of number of bands and fragments extracted (with lower molecular weight), while the use of the nondenaturing solution revealed a major intensity for most of the myofibrillar protein (with higher molecular weight) analysed.

In another work, M. Negera and A. P. Washe evaluate the potential of certain natural dietary spices, which are commonly used in food flavouring and preservation, to detoxify or degrade chemical carcinogens such as aflatoxin B1 (AFB1). The usage of natural plant extracts represents an interesting alternative to biological agents for aflatoxin detoxification as those would not alter significantly the nutritional properties of the product, plus its usage fits with a sustainable production of traditional food products. The reported findings revealed nonobvious benefits of the use of natural dietary spices as effective solution for AFB1 degradation and decontamination of food, providing scientific credit to the nonobvious benefits of indigenous knowledge of using natural spices for food flavouring and preservation to control aflatoxin.

Lastly, authentication of food products and detection of adulterations is particularly critical, given the difficulties to relate those with the concentration of specific compounds that can be assessed using classical analytical techniques. The application of pattern recognition or multivariate calibration methods allows one to infer underlying relationships between the measured analytical signals and the properties of the samples, approaches that are fundamental in food authentication.

In this regards, L. Du et al. propose the use of Fouriertransform infrared spectroscopy (FT-IR), in combination with chemometric methods such as principal component analysis (PCA) and partial least squares-discriminant analysis (PLS-DA), for the authentication of raw from reconstituted milk. To this purpose, the authors adulterated different raw milk samples with commercial milk powders at different levels, succeeding in the correct discrimination of raw and reconstituted milk samples.

In the same line, Tian et al. propose the combined use of an electronic tongue and an electronic nose for the detection of the adulteration of minced mutton with pork meat. To this aim, the authors made use of two commercial systems to measure the samples, and combined data were analysed by means of canonical discriminant analysis (CDA) and artificial neural networks (ANNs). The results showed that the authors were not only able to correctly discriminate both type of meats but also able to numerically predict the degree of adulteration (pork content).

\section{Conflicts of Interest}

The guest editors declare that they have no conflicts of interest regarding the publication of this special issue.

\section{Acknowledgments}

We thank the authors of the manuscripts included in this special issue for their contributions as well as all the reviewers for their valuable participation in the evaluation process.

Xavier Cetó

José M. Díaz-Cruz

Figen Tokatli

Paolo Lucci

Sabrina Moret 


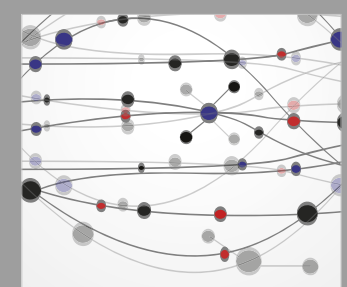

The Scientific World Journal
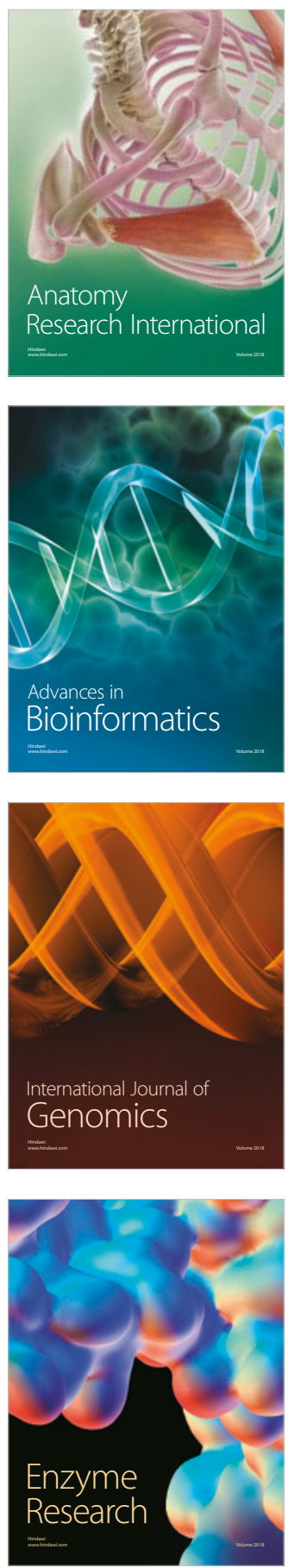
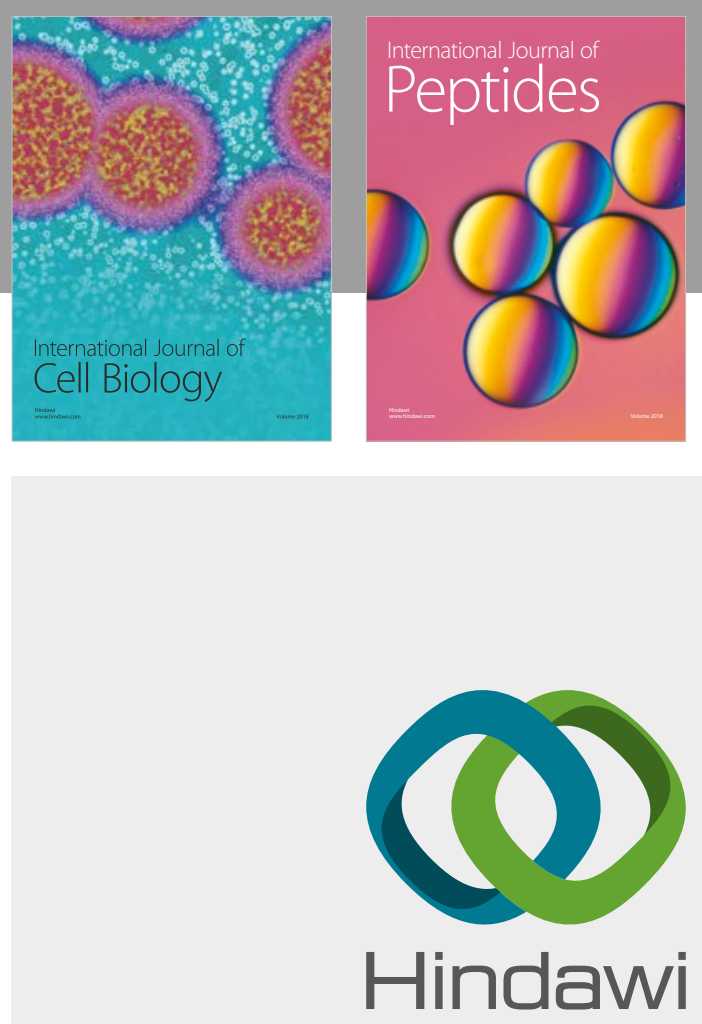

Submit your manuscripts at

www.hindawi.com
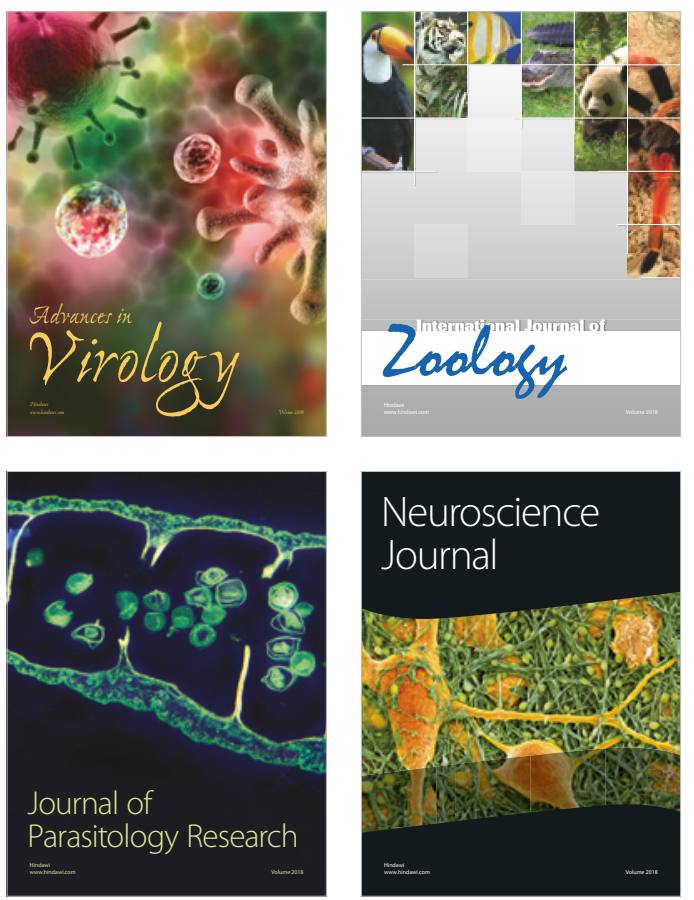
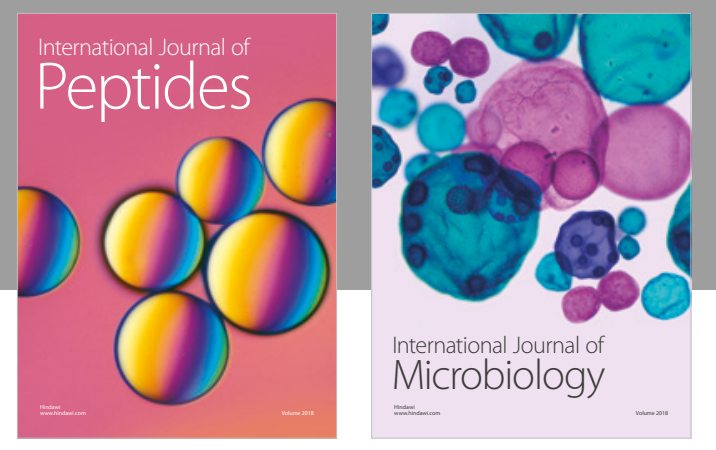

nternational Journal of Microbiology
Journal of
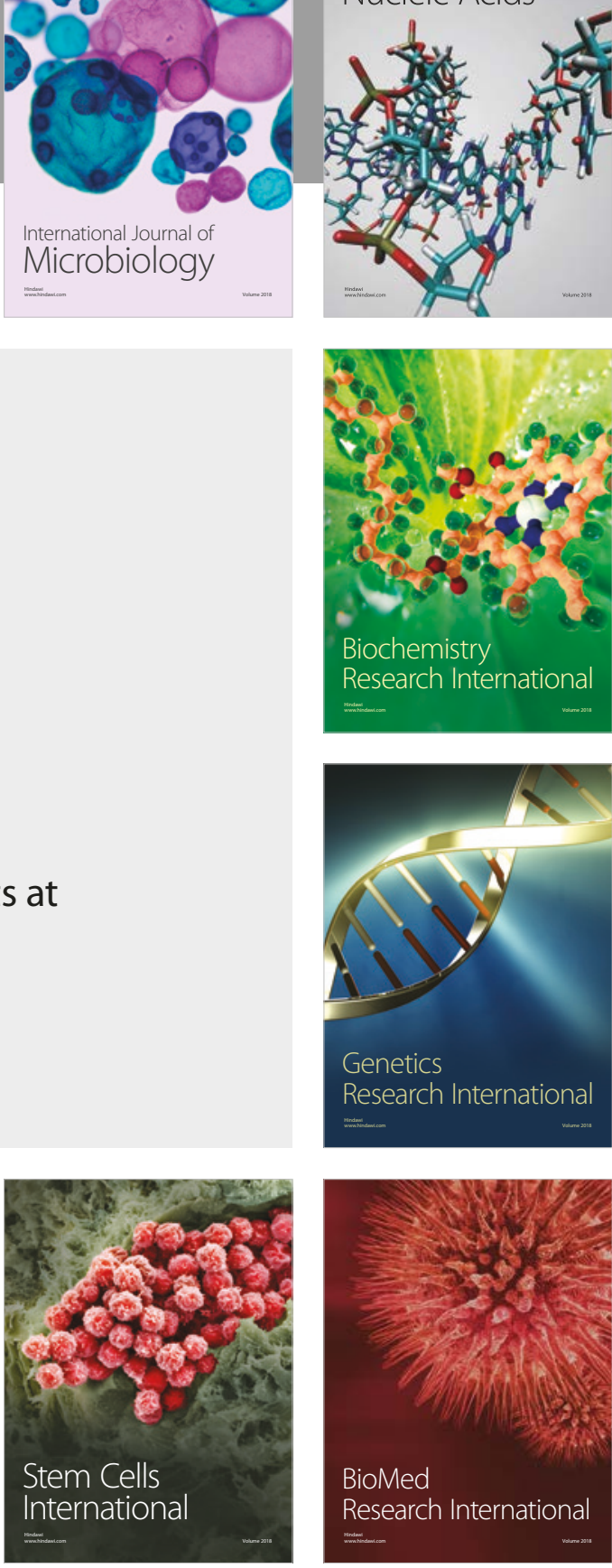
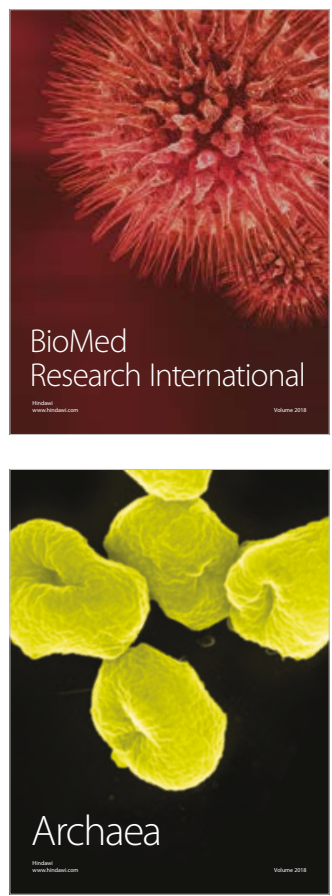